\title{
Correlación clínico-radiológica como primera ruta en el diagnóstico de síndrome de Gorham-Stout
}

Clinical-radiological correlation as the first route in the diagnosis of Gorham-Stout syndrome

\author{
Iván David Lozada-Martínez ${ }^{1}$ ORCID , Andrés Elías Llamas-Nieves ${ }^{10 R C I D}$, María Paz Bolaño-Romero' \\ ORCID, Sandra Herrera Lomonaco ${ }^{2}$ ORCID
}

Fecha correspondencia:

Recibido: febrero 21 de 2020.

Revisado: septiembre 7 de 2020.

Aceptado: octubre 20 de 2020.

Forma de citar:

Lozada Martínez ID, Llamas Nieves

AE, Bolaño Romero MP, Herrera

Lomonaco S. Correlación clínico-

radiológica como primera ruta

en el diagnóstico de síndrome de

Gorham-Stout. Rev CES Med. 2020;

34(3): 207-214.

Open access

(c) Derecho de autor

Licencia creative commons

Ética de publicaciones

Revisión por pares

Gestión por Open Journal System

DOl: http://dx.doi.org/10.21615/

cesmedicina.34.3.4

ISSN 0120-8705

e-ISSN 2215-9177
Comparte

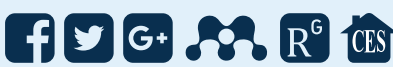

\section{Resumen}

El síndrome de Gorham-Stout constituye una rara enfermedad ósea cuya etiología sigue siendo desconocida, que se caracteriza por presentar osteólisis y proliferación anormal de canales vasculares. Se describe el caso de un paciente masculino de 47 años quien consultó por un cuadro de aproximadamente 15 días de evolución consistente en edema, dolor, eritema y secreción sero-hemática por herida quirúrgica en hombro derecho, con diagnóstico reciente de artritis séptica. Refería antecedente de síndrome de Gorham-Stout, evidenciándose al examen físico y radiografía comparativa de hombros. La presentación clínica de estos pacientes depende del área afectada y la extensión de la enfermedad, convirtiéndose su diagnóstico en un desafío en caso de que el deterioro se presente en zonas no expuestas. El principal síntoma consiste en dolor localizado, acompañado de hinchazón, debilidad y deterioro funcional de las extremidades afectadas. Estos pacientes pueden permanecer asintomáticos hasta sufrir una fractura ósea espontáneamente o después de un trauma menor.

Palabras clave: Osteólisis; Fracturas espontaneas; Enfermedades raras; Síndrome de Gorham-Stout.

\footnotetext{
Abstract

Gorham-Stout syndrome is a rare bone disease whose etiology remains unknown, characterized by osteolysis and abnormal proliferation of vascular channels. We describe the case of a 47-year-old male patient who consulted for 15 days of evolution of edema, pain, erythema and seroma expulsion from surgical wound in the right shoulder, with a recent diagnosis of septic arthritis. He referred a history of Gorham-Stout syndrome, evidenced with physical examination and comparative radiography of the shoulders. The clinical presentation of these patients depends on the affected area and the extent of the disease, making their diagnosis a challenge in case the deterioration occurs in unexposed areas. The main symptom is localized pain, accompanied by swelling, weakness and functional impairment of the affected limbs. These patients may remain asymptomatic until suffering a bone fracture spontaneously or after minor trauma.
} 


\section{Sobre los autores:}

1. Estudiante de Medicina, Universidad de Cartagena, Centro de Investigaciones Médico-Quirúrgicas.

\section{Medica Patóloga-}

Especialista en Gerencia en Seguridad Social. Jefe Laboratorio de Patología Clínica el Bosque. Jefe Laboratorio de Patología Hospital Naval de Cartagena Docente Facultad de Medicina Universidad de Cartagena.
Keywords: Osteolysis; Spontaneous fractures; Rare diseases; Gorham- Stout syndrome.

\section{Introducción}

El síndrome de Gorham-Stout constituye una rara enfermedad ósea con etiología desconocida, en la cual se presenta osteólisis y proliferación de los vasos linfáticos. Puede comprometer distintas regiones del esqueleto, tales como cráneo, región maxilofacial, brazos, costillas, pelvis, piernas y columna $(1,2)$. Se ha especulado que la proliferación anormal de los canales vasculares y linfáticos es producto de complejos mecanismos patogénicos que involucran factores de crecimiento endotelial vascular y actividad osteoclástica acelerada (3-5).

Aunque no presenta predilección por raza o edad, ocurre mayormente en niños y adultos jóvenes. Dada su rareza y presentación clínica variable, se ha convertido en un reto establecer oportunamente su diagnóstico (6).

A pesar de que el diagnóstico definitivo es histopatológico (7) existen datos positivos que permiten sospechar de la presencia de esta condición (8), y pueden obtenerse a través de una historia clínica detallada y posteriormente correlacionar con signos imagenológicos. Debido al escaso conocimiento de esta entidad y el número amplio de diagnósticos diferenciales a considerar, se realiza el siguiente reporte de caso y revisión de la literatura.

\section{Caso clínico}

Un paciente masculino de 47 años fue remitido a urgencias de un hospital de tercer nivel en la ciudad de Cartagena de Indias, por un cuadro de 15 días de evolución de edema, dolor, eritema y secreción serohemática por herida quirúrgica en hombro derecho, con diagnóstico reciente de artritis séptica. Como antecedentes de relevancia, refirió síndrome de Gorham, un trauma menor en hombro derecho seis meses previos al ingreso con luxo-fractura de humero. Dos meses previos al ingreso presentó luxación refractaria en la misma localización, por lo que le fue realizada reducción abierta con fijación interna. Un mes después se le realizó retiro de material y durante el postoperatorio, desarrolló el cuadro actual.

En el examen físico se encontró paciente en regulares condiciones generales, afebril, con limitación en movimiento de los brazos, además de signos de inflamación aguda y persistencia de secreción sero-hemática por herida quirúrgica en hombro derecho. Se realizó radiografía comparativa de hombros en donde se evidenció, en hombro derecho, fractura antigua diafisiaria distal de humero derecho con material de osteosíntesis, deformidad en la articulación glenohumeral, ausencia de cabeza humeral y edema de tejidos blandos (figura 1). En hombro izquierdo se observó imagen lítica a nivel de extremo proximal de humero y edema de tejidos blandos (figura 2). 
El síndrome de Gorham-Stout también es conocido como la enfermedad del hueso fantasma, enfermedad del hueso desaparecido, osteolisis masiva o resorción ósea espontánea aguda.

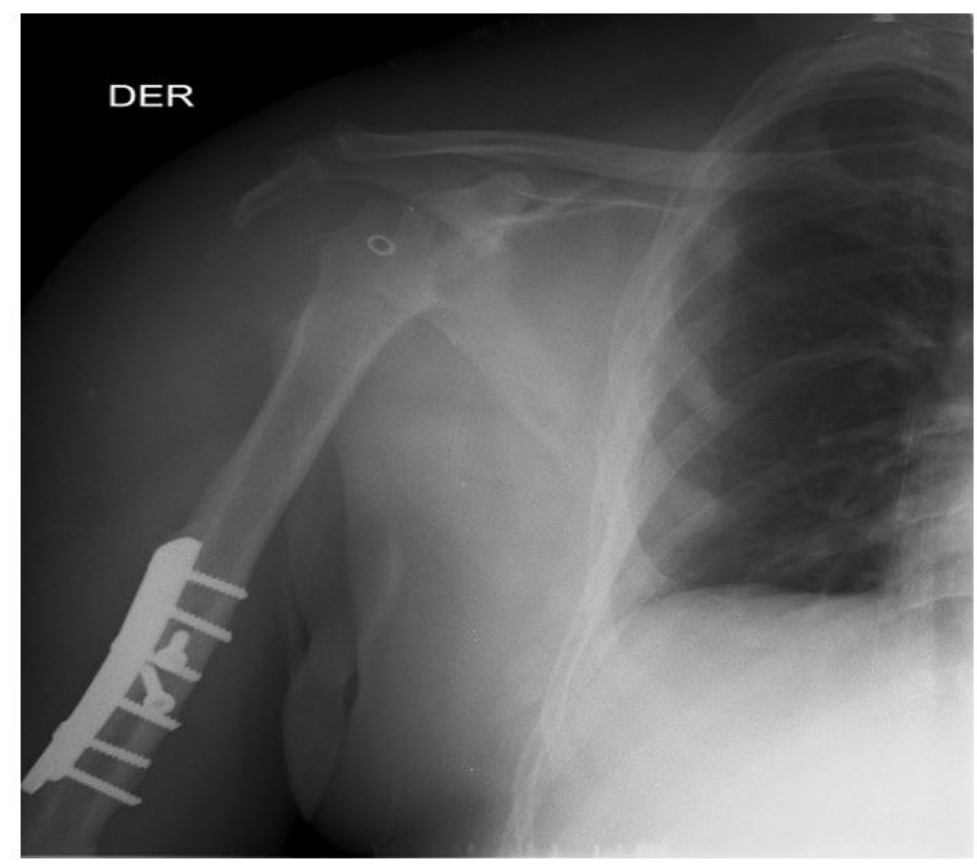

Figura 1. Radiografía de hombros comparativa. Se observa fractura antigua diafisiaria distal de humero derecho con material de osteosíntesis, deformidad en la articulación glenohumeral, ausencia de cabeza humeral y edema de tejidos blandos

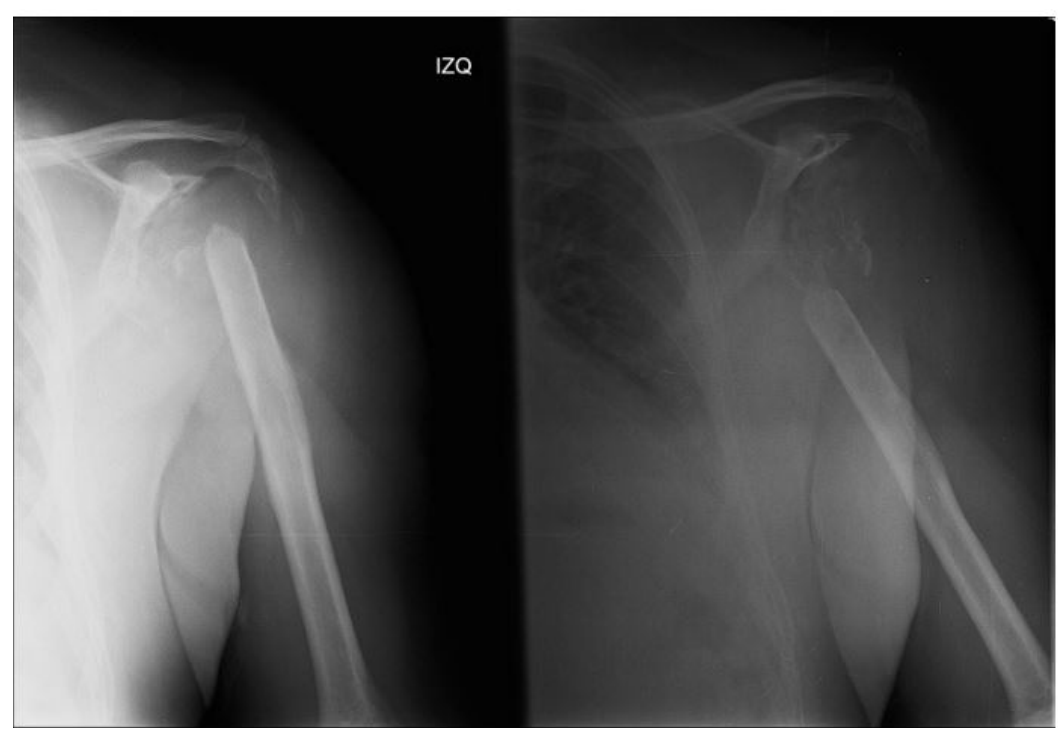

Figura 2. Radiografía de hombro derecho. Se observa imagen lítica a nivel de extremo proximal de humero izquierdo, y edema de tejidos blandos

En base a los signos observados durante el examen físico y el resultado de la ayuda imagenológica, se apoya la impresión diagnóstica de síndrome de Gorham y artritis séptica. Se establece antibioticoterapia por 72 horas, que debe luego suspenderse por respuesta tórpida, indicándose entonces lavado quirúrgico más desbridamiento, que se realiza un día después. Nuevamente, se instaura antibioticoterapia, observándose evolución satisfactoria y permitiendo el alta del paciente 10 días más tarde. 


\section{Discusión}

Esta entidad también es conocida como la enfermedad del hueso fantasma, enfermedad del hueso desaparecido, osteolisis masiva o resorción ósea espontánea aguda. Se caracteriza por una proliferación de canales vasculares y linfáticos en etapas tempranas de la enfermedad o hiperplasia fibrosa en etapas tardías (9).

La presentación clínica depende del área afectada y la extensión de la enfermedad, pudiendo ser irreconocible en caso de que el deterioro se presente en zonas no expuestas, convirtiéndose el diagnóstico en un desafío. A pesar de lo anterior, este se puede lograr a través del descarte de los diagnósticos diferenciales más frecuentes de osteólisis, tales como infecciones, cáncer, trastornos inflamatorios, metabólicos o endocrinos. El principal síntoma consiste en dolor localizado, acompañado de hinchazón, debilidad y deterioro funcional de las extremidades afectadas (10-13). Sorprendentemente, estos pacientes pueden permanecer asintomáticos hasta sufrir una fractura ósea espontáneamente o después de un trauma menor.

Brunner et al. reportan el caso de una paciente de 77 años quien acudió a emergencias por presentar un dolor en el hombro derecho, sin historia previa de trauma. Las radiografías demostraron una osteólisis progresiva rápida de la cabeza humeral y fractura patológica en la espina de la escápula derecha. Tras el análisis histopatológico de distintas muestras de hueso, no se evidenció rastros de infección o malignidad, por lo que se asumió la relación con el síndrome de Gorham-Stout (11).

Los diagnósticos diferenciales más frecuentes de osteólisis, son infecciones, cáncer, trastornos inflamatorios, metabólicos o endocrinos.
Los pacientes con compromiso torácico pueden consultar por dificultad para respirar. Wang et al. reportan el caso de una niña de 14 años quien presentó síndrome de Gorham con compromiso de columna vertebral en región torácica y quilotórax bilateral progresivo, el cual se gestionó con éxito gracias a la combinación de tratamientos conservadores y quirúrgicos (9). El compromiso vertebral puede causar graves defectos neurológicos, deformidades, parálisis y muerte. Un ejemplo de lo anterior es el caso reportado por Nozawa et al. en el que dicho compromiso se presentó en los huesos de la base del cráneo y de la región maxilofacial, concluyendo desafortunadamente en infarto del tallo cerebral secundario a la rápida osteólisis (1).

El diagnóstico se realiza teniendo en cuenta una historia clínica detallada del paciente, ayudas diagnósticas como rayos $X$, tomografía axial computarizada, resonancia magnética y el estudio histopatológico. El común denominador durante la revisión de la radiografía es la imagen lítica con deformidad del hueso y la presencia de fracturas antiguas (1,9,11), tal como se observó en este caso. Gran parte de los pacientes deben ser intervenidos quirúrgicamente, ya sea para la corrección de fracturas patológicas o para reconstrucción de huesos destruidos (5).

A pesar de que durante la evaluación del cuadro clínico ya se contaba con un diagnóstico previo en el paciente, se llevó a cabo una revisión exhaustiva en demás extremidades y regiones, donde no se encontró evidencia de trastornos óseos. Un punto importante a resaltar es la condición psicosocial del paciente. Refirió que su calidad de vida se veía notablemente afectada por la dificultad en la realización de actividades de la vida diaria, además de las complicaciones adheridas al trastorno, las cuales mencionó como dolorosas y extenuantes. Por lo que se recomienda remitir a terapia psicológica. 
Aunque no existe un tratamiento efectivo y definitivo, la radioterapia, los medicamentos anti osteoclásticos (bifosfonatos) y el interferón $a-2 b$, se han utilizado como opciones terapéuticas.
Aunque no existe un tratamiento efectivo y definitivo, la radioterapia, los medicamentos anti osteoclásticos (bifosfonatos) y el interferón $a-2 b$, se han utilizado como opciones terapéuticas. Se ha descrito a la ripamicina como un nuevo posible agente terapéutico, actuando como inhibidor de la activación de las células T mediante el bloqueo de la transducción de señales intracelulares dependientes e independientes de calcio, el cual ha mostrado inhibir la linfangiogénesis $(12,14,15)$.

\section{Conclusiones}

El síndrome de Gorham-Stout es una entidad de presentación excepcional, del cual se puede sospechar en función de una clínica relacionada con fracturas óseas de considerable magnitud, posteriores a un trauma menor. Lo anterior, relacionado a la evidencia de una imagen lítica de etiología desconocida en la imagenología, debe encender la alarma de solicitar un estudio histopatológico para realizar el diagnostico definitivo. Es necesario tener en cuenta que esta condición es inespecífica, puesto que su clínica depende de la severidad y región ósea afectada, por lo que siempre debe ser un diagnóstico diferencial en un cuadro de lesiones óseas recurrentes.

\section{Responsabilidades éticas}

\section{Protección de personas y animales}

Para esta investigación no se han realizado experimentos en seres humanos ni en animales.

\section{Confidencialidad de los datos}

Los autores declaran que han seguido los protocolos de su centro de trabajo sobre la publicación de datos de pacientes.

\section{Derecho a la privacidad y consentimiento informado}

Los autores declaran que en este artículo no aparecen datos de pacientes.

\section{Financiamiento}

No se recibió patrocinio de ningún tipo para llevar a cabo este artículo.

\section{Conflicto de intereses}

Los autores declaran no tener ningún conflicto de intereses.

\section{Bibliografía}

1. Nozawa A, Ozeki M, Hori T, Kato H, Ohe N, Fukao T. Fatal progression of Gorham-Stout disease with skull base osteomyelitis and lateral medullary syndrome. Intern Med. 2019; 58(13):1929-1933.

2. Jaccard A, Macedo C, Castro G, Guiroy A. Thoracic spine dislocation in Gorham-Stout Syndrome: Case report and literature review. Surg Neurol Int. 2018; 9:223.

3. Ruggieri P, Montalti M, Angelini A, Alberghini M, Mercuri M. Gorham-Stout disease: the experience of the Rizzoli Institute and review of the literature. Skeleton Radiol. $2011 ; 40(11): 1391-1397$.

4. Ozeki M, Asada R, Saito AM, Hashimoto H, Fujimura T, Kuroda T, et al. Efficacy and safety of sirolimus treatment for intractable lymphatic anomalies: a study protocol for an open-label, single-arm, multicenter, prospective study (SILA). Regen Ther. 2019; 10:84-91. 
5. Zanelli M, Zizzo M, Martino G, Bisagni A, De Marco L, Ascani S. Bone marrow biopsy disclosing a rare osteolytic disorder: Gorham-Stout Syndrome. Int J Surg Pathol. 2019; 28(1):76-77.

6. Maratib A, Maryam K, Syeda R, Laila T, Mohammad T. The first clinical case of Gorham-Stout Syndrome of humerus in an 18-year-old female reported in Pakistan. Cureus. 2019; 11(6): e4832.

7. Andrade RG, Sperb AP, Hochhegger B, Serafini OA, Valduga SG. Gorham-Stout syndrome: the radiologic-pathologic correlation as a diagnostic pathway when bone is vanishing. Radiol Bras. 2019; 52(2):131-133.

8. El-Kouba G, Santos RA, Pilluski PC, Severo A, Lech A. Síndrome de Gorham-Stout: "doença do osso fantasma". Rev Bras Ortop. 2010;45:618-22.

9. Wang P, Liao W, Cao G, Jiang Y. A rare of Gorham-Stout syndrome involving the thoracic spine with progressive bilateral chylothorax: a case report. BMC Musculoskeletal Disorders. 2019; 20:154.

10. Gulati U, Mohanty S, Dabas J, Chandra N. "Vanishing Bone Disease" in maxillofacial region: a review and our experience. J Maxillofac Oral Surg. 2015; 14(3):548-57.

11. Brunner U, Ruckl K, Konrads C, Rudert M, Plumhoff P. Gorham-Stout syndrome of the shoulder. SICOT J. 2016; 2:25.

12. Nikolaou VS, Chytas D, Korres D, Efstathopoulos N. Vanishing bone disease (Gorham-Stout syndrome): a review of a rare entity. World J Orthop. 2014; 5(5):694-698.

13. Kim BJ, Kim TH, Kim DJ, Noh D, Ham SJ, Lee S. A successfully treated case of Gorham-stout syndrome with sternal involvement. Korean J Thorac Cardiovasc Surg. 2015; 48(1):90-4.

14. Dellinger MT, Garg N, Olsen BR. Viewpoints on vessels and vanishing bones in Gorham-stout disease. Bone. 2014; 63:47-52.

15. Schell A, Rhee JM, Allen A, Andras L, Zhou F. Surgical management of Gorham disease involving the upper cervical spine with occipito-cervicalthoracic fusion: a case report. The Spine Journal . 2016; 16(7):e467-72. 